\title{
Optimal Traffic Re-Grooming Model for Heterogeneous Carrier Ethernet Services over WDM Optical Network.
}

\author{
Leandro Colombi Resendo, \\ Federal Institute of Espírito Santo
}

\begin{abstract}
This paper proposes three novel integer linear programming (ILP) formulations, where the first model deals with the Grooming, Routing, Wavelength Assignment, and Carrier Ethernet Interface Allocation Problem (GRWA-I); the second to Traffic Re-Grooming Problem (TRGP) to accommodate demand changes in a GRWA-I network scenarios; and the third to Traffic Re-Grooming demands in case of failures. The traffic re-grooming problem consists basically in assigning demands change in a working network, without affect others requests. In addition, in this work, it problem is formulated for the first time using ILP model. As TRGP needs a configured network scenario as input parameter, the first model proposed accomplish this task for the first network scenario. In numerical results, it was used a 14-node network (NSFnet) and 6 traffic matrix. Moreover, the models proposed were compared with a shortest path (SP) routing method. Results show the difference for network cost between an optimal method and a heuristic design over time, as well as the importance of an initial optimal configuration for future network growth.
\end{abstract}

Index Terms - Integer Linear Programming, Traffic Re-Grooming Problem, Carrier Ethernet, WDM Optical Network.

\section{INTRODUCTION}

Optical network has been well established as the technology to support the traffic demand in backbones, due to its capacity to support more than a hundred of channels by meaning of Wavelength Division Multiplexing (WDM), where each wavelength can transmit 100Gb/s. This technology has permitted the development of different corporative and domestic services, such as, Video on Demand (VoD), Cloud Services, Data Center, etc. The classical configuration problem in WDM optical networks supporting electronic processing is called traffic grooming, routing and wavelength assignment problem (GRWA). Because of its complexity to generate a good network configuration, this problem is frequently divided in smaller parts where the main goal is the optimization of resources [1]-[3]. The division for GRWA problem is made according these following sub-problems: Virtual Topology Design (VTD); Routing and Wavelength Assignment (RWA); and Traffic Grooming Problem (TGP)[1].

While WDM optical network has been consolidated as backbone physical layer, the data layer has a set of technological alternatives. Based in circuit switched paradigms, SONET/SDH was the earlier technological choice offering static, reliable, and bandwidth aggregated circuits. However, now, 
Carrier Ethernet has becoming a good option as backbone transport network technology, with features such as connection oriented services, cost, and better management functionality. Currently, to compose the transport network, the Carrier Ethernet interfaces has basically tree kind of interfaces, considering its bit rate, $10 \mathrm{~Gb} / \mathrm{s}, 40 \mathrm{~Gb} / \mathrm{s}$, and $100 \mathrm{~Gb} / \mathrm{s}$, standardized by IEEE[4]. As the Carrier Ethernet is implemented on the optical network, its interfaces are considered as demands that must be assigned on optical channels. Inspired from SDH-over-WDM, an important functionality found in Ethernet-over-WDM is the traffic grooming requests within optical channels, standardized in [5]. The main purpose of traffic grooming is optimizing the use of opto-electronic-opto interfaces, keeping the minimum number. The number of opto-electronic-opto transceivers is the main metric to evaluate the Capital Expenditure (CapEx) in a WDM network [6], and then its minimization has the goal of design a cost-effective network. The problem of assigning traffic demands on Carrier Ethernet and its devices on the WDM optical network is called traffic grooming, routing, wavelength assignment, and Carrier Ethernet interface allocation problem (GRWA-I). Considering the optical network constraints and different bit rate Carrier Ethernet interfaces, it is possible to find many works dealing with how to optimize the Carrier Ethernet interface assignment on WDM optical network. For instance, [7] proposes a heuristic to routing de $10 \mathrm{~Gb} / \mathrm{s}$ interfaces on wavelengths with capacity of $40 \mathrm{~Gb} / \mathrm{s}$; in [8], it was proposed an Integer Liner Programming (ILP) model to assign the Carrier Ethernet interfaces considering the traffic grooming problem and regenerators cost.

Besides of network design models, a recent important issue is how to keep the cost-effective network over time. The main idea on this question is developing strategies to accommodate the traffic evolution, where the most important constraint is to allocate new demands without interfere on the rest of previously set up requests. If a network has the traffic grooming functionality, such as optical network, it is called Traffic Re-Grooming Problem (TRGP). There are few investigations dealing with this issue [9]-[11], and then it still is considered a new problem in the literature. The three cited works propose heuristics for accommodate new demands minimizing the network cost, where a new request cannot affect the previously assigned demands.

Since the author has not found works presenting an ILP model dealing with TRGP, this work proposes the first ILP model to TRGP to Carrier Ethernet over WDM optical network. To test the model proposed it is necessary a network configuration where will be accommodate the new demands. Furthermore, there is also proposed another model with the task of configuring the Carrier Ethernet interfaces on an optical network, considering an initial traffic matrix. To both models, the objective function used is minimizing the number of Carrier Ethernet interfaces. The functionality of keep previous demand and optimize a network change may offer plenty kind of reconfigurations tests. In addition, it was proposed a modification in Re-Grooming ILP model to reassign demands or the traffic engineering to reconfiguration for predicted failures. As study case, the traffic re-grooming model proposed was compared with a routing method using the shortest path strategy, and computational experiments are accomplished with 6 increasing traffic matrices on a 14-node network. 
The remainder of this paper is as follows. The next section presents characteristics of network scenarios considered and models proposed, one formulation to generate the optimal network configuration, one to TRGP for traffic evolution, and a model to TRGP minimizing the number demands that can not be reassigned in case of failure. Section III shows the numerical results achieved by models, and as a comparison, it is presented results achieved by configuration accomplished by shortest path process. It process is widely used as routing strategy in commercial devices and protocols. In addition, the results simulate the growth of demands by mean of 6 traffic matrices. The conclusion and final considerations are presented in IV.

\section{PROBLEM FORMULATION}

The proposed model consider a mesh network with opaque nodes and two fiber links between each pair of nodes $i$ and $j$ (represented by $i j$ and $j i$ ), where every link supports the same amount of wavelength. With a set of node, lightpaths, and traffic demands as inputs, the model deals with GRWA-I using as objective function minimizing the cost with Carrier Ethernet interfaces. Moreover, it is permitted a heterogeneous use of Carrier Ethernet interfaces with $10 \mathrm{GbE}, 40 \mathrm{GbE}$ and $100 \mathrm{GbE}$, where these interfaces/services are accommodated on optical channels with 100Gb/s of capacity. The electronic traffic grouping allows that different client bit rate interfaces could be conveniently groomed on optical layer. For example, let a demand of $20 \mathrm{~Gb} / \mathrm{s}$ between two nodes (where can be accomplished with two $10 \mathrm{GbE}$ interfaces or one $40 \mathrm{GbE}$ ), then it can be electronically processed and inserted with other demands to an effective used of a $100 \mathrm{~Gb} / \mathrm{s}$ optical channel.

Offering more flexibility, it was assumed that a request can be divided in different routes, respecting the constraint that the division must be done only in the source node and each part must keep the kind of interface along with the route [5]. The following subsections present the models proposed: first model deals with the optimization of demands routing on Carrier Ethernet Interfaces and wavelength assignment, minimizing the CapEx (cost with interfaces); the second approach receive a working network scenario as input, accomplishing the new demands allocation keeping the remainder unchanged, and minimizing the cost with new interfaces; and third model proposes an approach to traffic recovery in case of failures, aiming minimize the traffic lost it model use the surplus network capacity to try recovery the traffic lost in case of failures.

\section{A. ILP Model to Grooming, Routing, Wavelength, and Client interfaces assignment (GRWA-I)}

The first model presents as result an optimal network configuration containing the routes, wavelength and interfaces used by each demand. As following shown, this model is used to generate the input parameters to re-grooming formulation. In addition, its objective function is to minimize the network CapEx, represented by number of Carrier Ethernet interfaces. Fig. 1 illustrates the functionality offered by a considered node. 


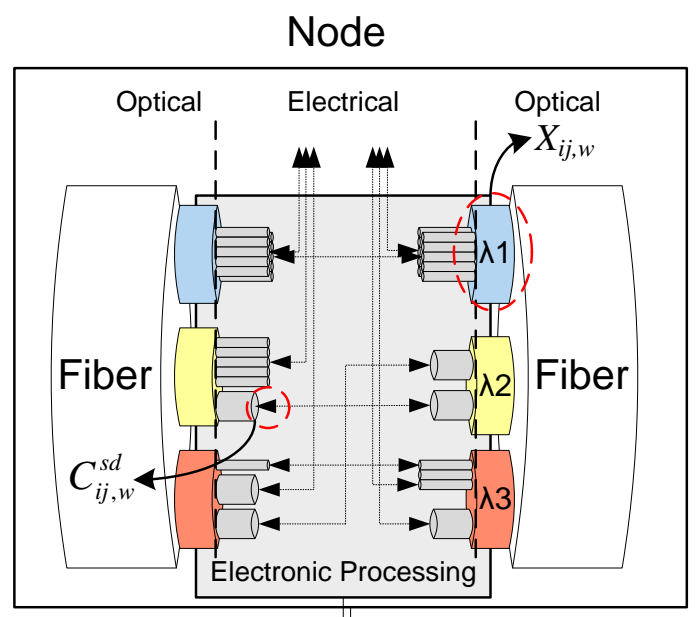

Fig. 1 An illustration for traffic grooming [12]

Fig. 1 shows the optical channel Transport Unit capacity considered, OTU4, that can support until $100 \mathrm{~Gb} / \mathrm{s}$ Carrier Ethernet services. As mentioned, each signal is electronic processed and must keep the same kind of interface along with the path. On wavelength $\lambda 1$ is illustrated a set of 10xODU2, where part is processed in the represented node and part is added and dropped at node. To illustrate the grooming capacity, wavelengths $\lambda 2$ and $\lambda 3$ show some possibilities, such as: in $\lambda 2,5 x O D U 2$ is dropped and 1xODU3 is electronically processed; in $\lambda 3,1 \mathrm{xODU} 3$ is added and dropped, and ODU2s are added, dropped, and electronically processed; moreover, in $\lambda 2$ and $\lambda 3$ is illustrated a demand that offered a wavelength conversion, by mean of electronic processing.

The following notation is used in our mathematical model:

$i$ and $j$ : are network nodes, bearing fiber link $i j$ (represented by $i j$ and $j i$ ).

$s$ and $d$ : are source and destination nodes respectively.

$w$ : is a wavelength in a link $i j$.

$k=\{10,40,100\}$ : is a variable index representing $10 \mathrm{~Gb}, 40 \mathrm{~Gb}$, and $100 \mathrm{~Gb}$ Carrier Ethernet interfaces.

\section{Given:}

$E[i][j]$ : the adjacency matrix.

$N$ : number of nodes in the network.

$\operatorname{traf}[s][d]$ : traffic matrix.

Cap: traffic capacity of a wavelength.

Cust ${ }^{k}$ : relative cost of each kind of interface $k$.

$\delta_{i j, w}^{s d}$ : Link-Path indicator. It is a binary that is set in case link $i j$ is used in route $r$ to meet demand $s d$, and zeroed otherwise. It is provided by the Yen's Algorithm.

\section{Variables:}

$C_{r, w}^{s d, k}:$ is the amount of traffic in wavelength $w$ and interface $k$ using route $r$ from source $s$ to destination $d$. It is illustrated in Fig. 1. 
$X_{i j, w}$ : is the amount of traffic in link $i j$ on wavelength $w$. It is illustrated in Fig. 1.

$X B_{i j, w}$ : is a binary variable indicating the use of a wavelength in a link, and equals one if the wavelength $w$ is used at link ij and zero otherwise.

$I_{i j, w}^{k}$ : indicates the quantity of $k$-interface used in the link $i j$ on wavelength $w$.

Objective Function: minimize the cost with Carrier Ethernet interfaces required to satisfy all the traffic demands.

$$
\text { Min: } \sum_{k} \sum_{i} \sum_{j} \sum_{w} C u s t^{k} \times I_{i j w}^{k}
$$

\section{Constraints:}

$$
\begin{gathered}
\sum_{w} \sum_{k} \sum_{r} C_{r, w}^{s d, k}=\operatorname{traf}[s][d], \forall_{s, d} \\
\sum_{s} \sum_{d} \sum_{r} \delta_{i j, r}^{s d} \times C_{r, w}^{s d, k} \leq k \times I_{i j, w}^{k}, \forall k, i, j, w \\
\sum_{k} k \times I_{i j, w}^{k}=X_{i j, w}, \forall_{i, j, w} \\
\operatorname{Cap} \times X B_{i j, w} \geq X_{i j, w}, \forall_{i, j, w}
\end{gathered}
$$

\section{Description:}

Expression (2) ensures that all the traffic between each pair of node $s d$ will be assigned. In addition, this constraint allows that a demand can be carried by multiple routes and channels, however it limits this division must occur only in the source node.

Expression (3) restrains the quantity of traffic in an interface to its capacity.

Expression (4) shows that $X_{i j, w}$ is equals the sum of every Carrier Ethernet Interface assigned in this channel. It represents the grooming of Carrier Ethernet Interface on Optical Network.

Expression (5) has two goals, first to create a relation between variables $X_{i j, w}$ and $X B_{i j, w}$, and second assuring that the quantity of traffic on a wavelength never exceed its capacity. In addition, the variable $X B_{i j, w}$ can be used to count the number of transceivers in the optical layer, and employed as objective function. Nevertheless, this goal is out of this work's scope.

\section{B. ILP model to Traffic Re-Grooming Problem (TRGP)}

This model has as input a working network scenario and the traffic (or network) alterations. It must be highlighted that it is the first ILP model that consider a working network state as an input problem. Since the idea of network optimization is kept in TRGP, every parameter and constraint employed in GRWA-I model also will be used here. Moreover, TRGP requires adding the following parameters and constraints.

\section{Parameters:}

$\mathrm{R}$ : set of new demands that most be assigned.

$N e t_{r, w}^{s d, k}$ : bears the working set-up for routing, wavelength assignment, and Carrier Ethernet Interface used for each demand $s d$. 
NetI ${ }_{i j, w}^{k}$ : contain the information about the number and type of interfaces used in each optical channel.

\section{Constraints:}

$$
\begin{gathered}
C_{r, w}^{s d, k}=N e t_{r, w}^{s d, k}, \forall s, d \notin R \\
I_{i j, w}^{k} \geq \operatorname{NetI}_{i j, w}^{k}, \forall k, i, j, w
\end{gathered}
$$

\section{Description:}

Expression (6) loads the working network configuration excluding the demands affected by traffic growth. Additionally, this constrains set part of decision variables reducing the model complexity.

Expression (7) ensures that the new network configuration will have at least the same number of interfaces in each node. With this constraint the new network configuration will prioritize the use of set up resources, avoiding an interface position change.

\section{Optimal Recovering-Oriented Configuration Design}

This model is constrained by the available (deployed minus faulty) resources and aims at traffic engineering protection set-ups with the largest amount of recovered traffic. Besides parameters and constraints presented in Subsections A and B, to this model requires adding the following parameters and variables.

\section{Parameters:}

$F$ : contains the demands $s d$ in need of rerouting (e.g. due to node, link, or wavelength failure) that will be dealt with by the traffic engineering protection arrangements.

\section{Variables:}

$D^{s d}$ : is a slack variable with the traffic $s d$ that can not be reassigned.

$S^{s d}$ : is the fraction of traffic $s d$ that can be reassigned.

$I B_{i j, w}^{k}$ : is a binary 1 if the interface $k$ on wavelength $w$ at link $i j$ was not affected by the failure, and zero otherwise.

Objective Function: As the network is configured, the goal is minimize the unattended demands.

$$
\text { Min: } \sum_{s} \sum_{d} D^{s d}, \forall s, d \notin F
$$

\section{Constraints:}

$$
\begin{gathered}
C_{r, w}^{s d, k}=N e t_{r, w}^{s d, k}, \forall s, d \notin F \\
D^{s d}+S^{s d}=\operatorname{traf}[s][d], \forall_{s, d} \\
\sum_{w} \sum_{k} \sum_{r} C_{r, w}^{s d, k}=S^{s d}, \forall_{s, d} \\
I B_{i j, w}^{k}=\left\{\begin{array}{l}
1 ; \text { when } \sum_{s d \notin F} N e t_{r, w}^{s d, k} \neq 0 \\
0 ; \text { otherwise }
\end{array}\right.
\end{gathered}
$$




$$
I_{i j, w}^{k}=N e t I_{i j, w}^{k} \times I B_{i j, w}^{k}, \forall k, i, j, w
$$

\section{Descriptions:}

Expression (9) is similar to (6), where the difference is that in (6) $R$ contains the new demands while $F$ is a set of demands that must reassigned.

Expression (2) in GRWA-I model is here replaced by (10) and (11). Expression (10) divide a demand of traffic in two parts, the reassigned demands, $S^{s d}$, and the demands that can not be reassigned, $D^{s d}$. It ensures that traffic demand $s d$, whether recovered or not, is bounded by the demand matrix (traf $[s][d])$. As $D^{s d}$ is minimized, the Expression (11) reassigns the maximum quantity of demands.

Expression (12) set the variable $I B_{i j, w}^{k}$ with 1 if the interface $k$ on wavelength $w$ at link $i j$ was not affected by the failure, 0 otherwise.

Expression (13) use the variable $I B_{i j, w}^{k}$ to limit the devices used. It loads the interfaces set from the network configuration, i.e., there is only available interfaces (on wavelength $w$ ) at links $i j$ that are crossed by an unaffected demands $s d$.

\section{NUMERICAL RESULTS}

To evaluate the TRGP model it was accomplished a numerical comparison with Shortest Path (SP) routing strategy, a well-known commercially used algorithm. For computation tests has been used a 14-node network (NSFnet), shown in Fig. 2, under 6 traffic matrices. The CPLEX Optimization Studio Academic Research 12.3 was used to solve the ILP formulation. The model used to generate the initial scenario, GRWA-I, needed of 40 minute of processing to achieve an optimal solution. However, due to complexity reduction in TRGP model, the new traffic demands were optimal allocated in 10 minutes to every traffic increase.

The initial traffic scenario was generated with a full traffic matrix and demands between $10 \mathrm{~Gb} / \mathrm{s}$ and $100 \mathrm{~Gb} / \mathrm{s}$, uniformly distributed, considering $10 \mathrm{~Gb} / \mathrm{s}$ as traffic granularity. To analyze the demands evolution, from the initial matrix, 5 others were interactively generated. From initial matrix, $5 \%$ of demands are selected to duplicate their traffic requests. It process was repeated 5 times, always under the last matrix generated.

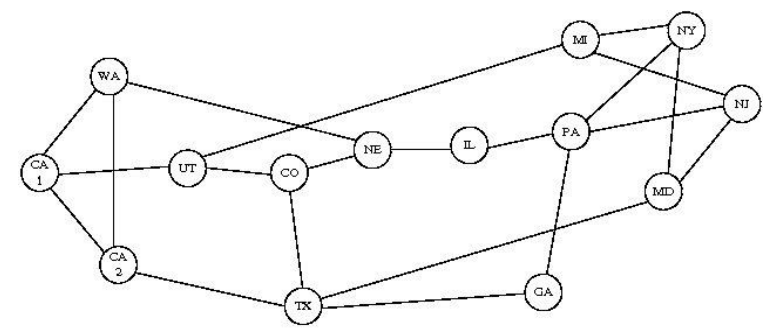

Fig. 2 NSFnet 
As presented in the objective function, Expression (1), the metric used to compare results and measure the model performance is the cost with Carrier Ethernet interfaces, which represent the layer 2 CapEx. Due to the difficult to obtain accurate information about values for each kind of interface, it was considered a relative cost to each type of interfaces. Inspired in [8], the interface cost of $10 \mathrm{~Gb}$ systems is fixed with an unitary value, while $40 \mathrm{~Gb}$ interface is the double of the 10Gb interface value, and the $100 \mathrm{~Gb}$ interface is the double of the $40 \mathrm{~Gb}$ cost.

Fig.s 3 and 4 present the cost achieved by re-grooming proposed model (TRGP) and shortest path routing (SP). In Fig. 3, TRGP and SP strategies were used over two initial scenarios, generated by GRWA-I (a) and SP (b).

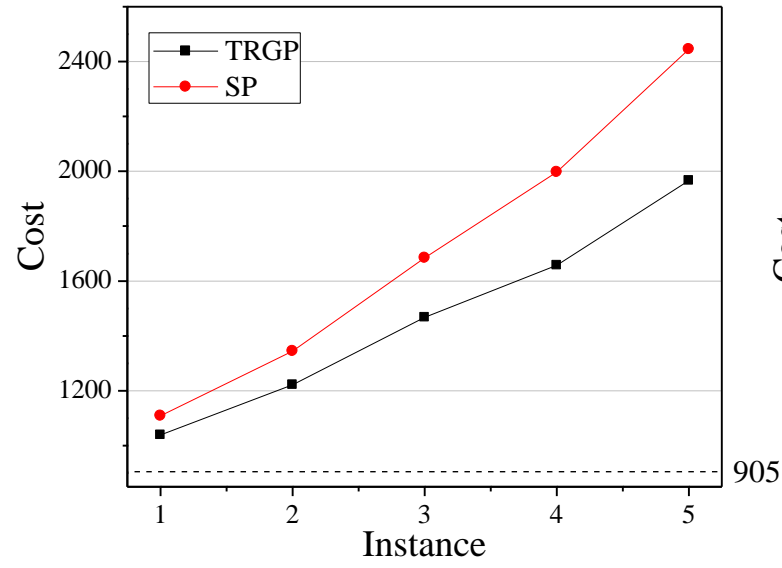

(a)

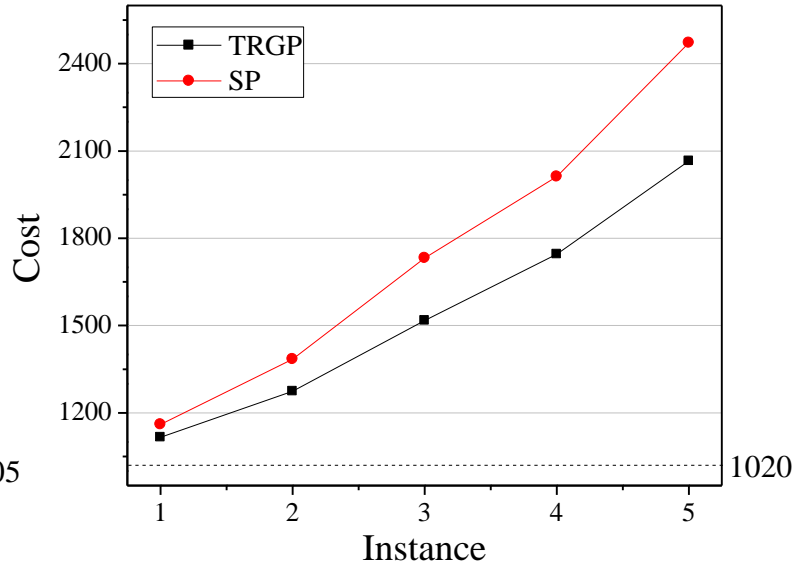

(b)

Fig. 3 TRGP and SP results, achieved by an optimized initial scenario, GRWA-I, (a) and heuristic initial scenario, SP, (b).

In Fig. 3 (a) and (b), the dotted line shows the cost for initial scenarios achieved by the methods GRWA-I (a) and SP (b). As expected, the proposed model attained betters results as compared with SP. For instance, in initial scenario the difference between the two methods was 115 (1020 - 905) unity of cost. However, different from expected, in the first two re-grooming iterations both methods achieved similar values. This result shows that for two iteration of traffic chance, optimal and heuristic strategies can obtain similar results for both initial scenarios, optimized or not. Meanwhile, after successive changes the TRGP advantages becomes clear, thus for long-term it is obvious the necessity of using an efficient optimization process to routing the new network demands.

Fig. 4 shows the TRGP model applied in two initial scenarios, generated by GRWA-I model ( $\mathbf{\Delta})$ and heuristic SP $(\nabla)$. Abscise shows the instances, for example the abscise 0 presents the results achieved by two strategies applied on the same initial matrix. Different from expected, the TRGP model cannot equalize the interface costs with the traffic growth, even after five interactions. It shows that, still being a model to obtain optimal configurations, TRGP cannot eliminate the difference imposed by a bad initial configured network. This result makes clear the necessity of efficient initial network scenario. 


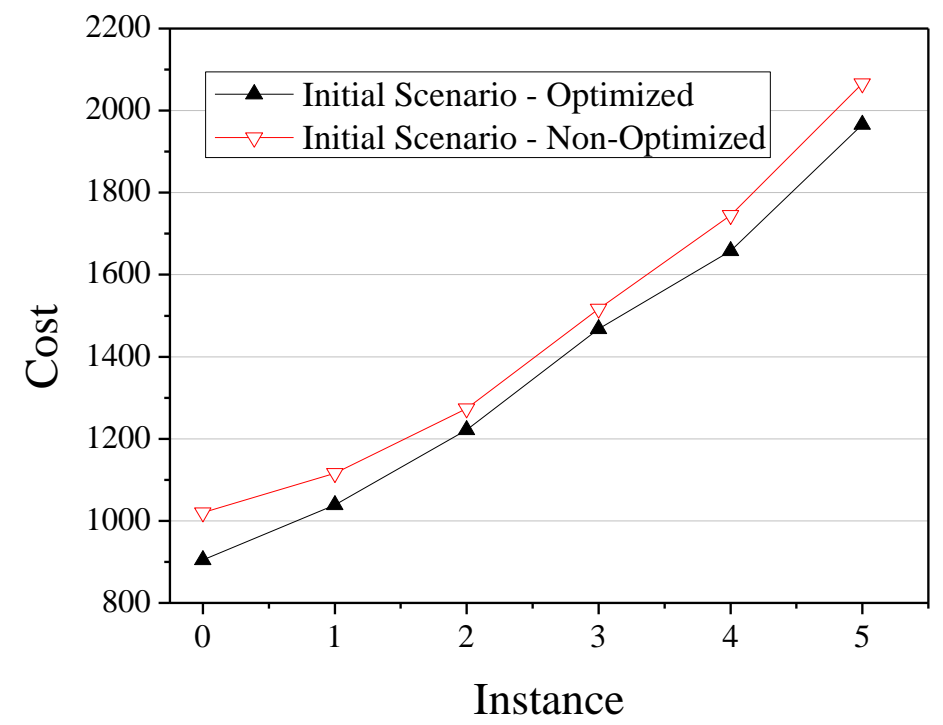

Fig. 4 TRGP results achieved by two initial scenarios, optimized and Non-Optimized.

Besides the cost, it was also evaluated the number and type of Carrier Ethernet Interface used in each scenario achieved. Table I shows the quantity of each kind of interfaces used to re-grooming configuration, through TRGP and SP strategies, on initial scenario generated by GRWA-I model. In instance 0 , it is noted that GRWA-I presents a $100 \mathrm{Gbs}$ interfaces predominance, however some $10 \mathrm{Gbs}$ and $40 \mathrm{Gbs}$ also were used avoiding unneeded costs. It is explained by the relation between relative cost for each kind of interfaces and its capacity. In addition, this feature also is observed in other instances optimized by TRGP method. On the other hand, in SP strategy is observed an exclusive choice of $100 \mathrm{Gbs}$ interfaces. As a consequence, it is noted that shortest path strategy generates network scenarios with underused resources. For example consider the instance 5: TRGP set up a network with $46300 \mathrm{Gbs}(100 * 434+40 * 69+10 * 14)$ of total capacity, while the network set up by SP has $60990 \mathrm{Gbs}(100 * 606+40 * 9+10 * 3)$ of total capacity, both for the same traffic matrix.

TABLE I. QUANTITY AND TYPE OF EACH INTERFACE USED

\begin{tabular}{|c|c|c|c|c|c|c|}
\cline { 2 - 7 } \multicolumn{1}{c|}{} & \multicolumn{5}{c|}{ GRWA-I } \\
\hline Instance & \multicolumn{2}{c|}{$\mathrm{k}=10$} & \multicolumn{2}{c|}{$\mathrm{k}=40$} & \multicolumn{2}{c|}{$\mathrm{k}=100$} \\
\hline 0 & \multicolumn{2}{|c|}{3} & \multicolumn{2}{c|}{9} & \multicolumn{2}{c|}{221} \\
\hline & \multicolumn{3}{c|}{ TRGP } \\
\hline Instance & $\mathrm{k}=10$ & $\mathrm{k}=40$ & $\mathrm{k}=100$ & $\mathrm{k}=10$ & $\mathrm{k}=40$ & $\mathrm{k}=100$ \\
\hline 1 & 9 & 25 & 244 & 3 & 9 & 272 \\
\hline 2 & 10 & 38 & 281 & 3 & 9 & 332 \\
\hline 3 & 10 & 41 & 340 & 3 & 9 & 416 \\
\hline 4 & 14 & 54 & 379 & 3 & 9 & 494 \\
\hline 5 & 14 & 69 & 434 & 3 & 9 & 606 \\
\hline
\end{tabular}

\section{CONCLUSION}

This work proposes three ILP models, where the first has the goal of achieve the optimal configuration considering the assignment of demand on two layers, Carrier Ethernet over optical 
layer; second, to find the optimal re-grooming where the foremost characteristic is keeping unchanged the working demands; and third, an optimal re-grooming approach to recover the maximal demands lost in case of failure. The TRGP was thought to bear any kind of network change, but in spite of the TRGP flexibility, the numerical experiments it was focused on traffic growth to compare with another re-grooming process found in the literature, shortest path. In addition, Carrier Ethernet Interfaces minimization was used as objective function, different from number of transceivers that is frequently found in literature. Due to TRGP model flexibility, it was adapted for a model to design the traffic reassignment on failure cases or in any other network disturb. Different from two models before presented, instead of minimize the cost with interfaces here it number is kept and it was minimized the quantity of demands unattended after a failure.

In numerical experiments it was used a 14-node network (NSFnet) and 6 traffic matrix, analyzing the traffic growth on the network. From an initial traffic matrix, the next traffic matrix is generated duplicating $5 \%$ of traffic requests, which were randomically selected. This experiment shown that for two iteration of traffic chance, optimal and heuristic strategies can obtain similar results for both initial scenarios, however to next iterations is observed the difference between these two methods, highlighting the TRGP effectiveness. For the second experiment, the TRGP was applied to two initial scenarios, generated by GRWA-I and SP, showing the difficulty of TRGP equalize these two scenarios. This experiment demonstrates the importance of a well designed initial scenario for the future network evolution. As shown in Fig. 4, even an optimal approach cannot mitigate the cost difference between an optimal (GRWA-I) and a rough (SP) initial scenario. In addition, as expected, due to relative cost used, it was observed a predominance of $100 \mathrm{Gbs}$ interfaces for both methods, however the shortest path routing strategy tends to produce an underused network scenarios.

\section{REFERENCES}

[1] R. Ramaswami e K. N. Sivarajan, Optical Networks: A Practical Perspective. Morgan Kaufmann, 2002.

[2] L. C. Resendo, L. C. Calmon e M. R. N. Ribeiro,Simple ILP Approaches to Grooming, Routing, and Wavelength Assignment in WDM Mesh Networks, SBMO/IEEE MTT-S, International Conference of Microwave and Optoelec-tronics, Brasilia, p.616-619, 2005.

[3] Q. D. Ho e M. S. Lee; Traffic Grooming in Large Optical WDM Mesh Networks with Wavelength Conversion Capability., Communications, 2006. ICC '06. IEEE International Conference on, pp. 2427 - 2432, Jun. 2006

[4] IEEE Std 802.3ba, Amendment 4: Medium access control parameters, physical layers and management parameters for 40Gb/s and $100 \mathrm{~Gb} / \mathrm{s}$ operation, June 2010.

[5] ITU-T Recommendation G.709 (2009).

[6] A. Morea e J. Poirrier, A critical analysis of the possible cost savings of translucent networks, in Proc.DRCN 2005, pp. 311-317.

[7] M. Batayneh, et al., Cost-Efficient Routing in Mixed-Line-Rate (MLR) Optical Networks for Carrier-Grade Ethernet, OFC/NFOEC 2008, OThB2, 2008.

[8] J. Santos, et. al., Scalable and Cost-Effective of Design of Optical Transport Networks Supporting Heterogeneous Ethernet Services, ECOC 2010, pp. 19-23, September, 2010.

[9] A. Kadohata, et al, Multi-Layer Greenfield Re-Grooming with Wavelength Defragmentation, Communications Letters, IEEE, pp 1-3, Feb. 2012.

[10] M. Fukutoku, et al, Optimized multi-layer optical network using in-service ODU/wavelength path re-grooming, Optical Fiber Communication Conference and Exposition (OFC/NFOEC), 2011 and the National Fiber Optic Engineers Conference, pp. 1-3, Jun. 2011.

[11] V. Hutcheon, OTN to enable flexible networks, Optical Fiber Communication Conference and Exposition (OFC/NFOEC), 2011, pp. 13, Jun. 2011.

[12] M. Tomizawa, et al., Photonic Network Node Technology for Transparent Path Accommodation, NTT Technical Review, Vol. 9, No. $8,2011$. 